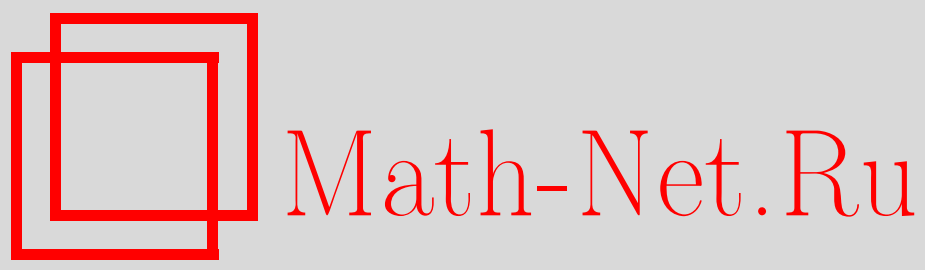

В. М. Бухштабер, В. З. Энольский, Явное алгебраическое описание гиперэллиптических якобианов на основе $\sigma$-функций Клейна, Функи. анализ и его прил., 1996, том 30, выпуск 1, 57-60

DOI: https://doi.org/10.4213/faa505

Использование Общероссийского математического портала MathNet.Ru подразумевает, что вы прочитали и согласны с пользовательским соглашением

http://www. mathnet.ru/rus/agreement

Параметры загрузки:

IP : 3.93 .64 .190

26 апреля 2023 г., $17: 45: 48$

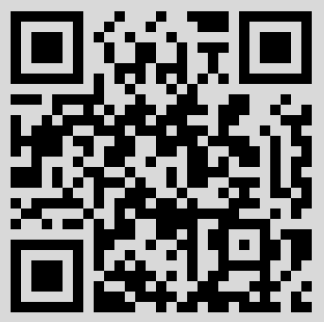




\section{Явное алгебраическое описание гиперэллиптических якобианов на основе $\sigma$-функций Клейна}

(c) 1996. В. М. Бухштабер, В. З. Энольский ${ }^{1}$

Задача алгебраического построения якобиана гиперэллиптической кривой рода $g$ хорошо известна. Необходимый для ее решения аппарат был развит уже в основополагающей работе Клейна [1] (подробности можно найти в [2]). Однако, насколько известно авторам, явные формулы не были тогда получены, и развитый аппарат впоследствии не использовался.

Под влиянием современных достижений в теории вполне интегрируемых динамических систем Мамфорд вновь рассмотрел этот вопрос. Предложенное им решение [3] основано на рекуррентной процедуре Маккина и ван Мёрбеке [4]. Следует отметить, что из результатов пионерских работ Новикова, Дубровина, Итса и Матвеева (см. обзор [5]), выполненных ранее [4], следует, что гиперэллиптические якобианы реализуются как линии уровня полиномиальных интегралов стационарных высших уравнений КдВ.

В этой заметке, опираясь на классическую теорию гиперэллиптических $\sigma$-функций Клейна [1], мы даем явное алгебраическое описание якобиана гиперэллиптической кривой рода $g$ и соответствующего многообразия Куммера.

Это исследование инициировано недавней работой авторов [6], где на основе $\sigma$-функций Клейна были построены явные решения важных уравнений в частных производных; дальнейшему развитию этих результатов посвящена работа [7].

Пусть

$$
V=\left\{(y, x) \in \mathbb{C}^{2}: y^{2}=4 x^{2 g+1}+\sum_{i=0}^{2 g} \lambda_{2 g-i} x^{2 g-i}\right\}
$$

- гиперэллиптическая кривая рода $g$, снабженная каноническим базисом циклов $\left(A_{1}, \ldots, A_{g} ; B_{1}, \ldots, B_{g}\right)$, т. е. базисом в группе одномерных гомологий $H_{1}(V, \mathbb{Z})$ с матрицей пересечения $\left(\begin{array}{cc}0 & -\mathbb{I}_{g} \\ \mathbb{I}_{g} & 0\end{array}\right)$, где $\mathbb{I}_{g}$ есть единичная $g \times g$ -
матрица.

Пусть $\left\{d u_{k}\right\}_{k=1}^{g}, d u_{k}=x^{k-1} d x / y, k=1, \ldots, g$, - канонические голомор $\Phi-$ ные дифференциалы в $\mathscr{H}^{\mathcal{A}}(V, \mathbb{C})$ и $2 \omega=\left(\oint_{A_{k}} d u_{l}\right), 2 \omega^{\prime}=\left(\oint_{B_{k}} d u_{l}\right)$ - невырожденные $g \times g$-матрицы их $A$ - и $B$-периодов. Под действием преобразования $(2 \omega)^{-1}$ в $\mathscr{H}^{\mathbb{A}}(V, \mathbb{C})$ вектор $d u^{T}=\left(d u_{1}, \ldots, d u_{g}\right)$ отображается в вектор нормированных голоморфных дифференциалов $d v^{T}=\left(d v_{1}, \ldots, d v_{g}\right)$, т. е. в вектор из $\mathscr{H}^{A}(V, \mathbb{C})$, компоненты которого нормированы условиями $\oint_{A_{k}} v_{l}=\delta_{k l}$, $k, l=1, \ldots, g$. Известно, что $g \times g$-матрица $\tau=\left(\oint_{B_{k}} v_{l}\right)=\omega^{-1} \omega^{\prime}$ принадлежит

\footnotetext{
1Эти исследования были частично финансированы грантами M3Z000 и U44000 Международного научного фонда и грантом 94-01-01444 РФФИ.
} 
верхнему полупространству Зигеля $\mathscr{S}_{g}$ степени $g$, т. е. является симметрической матрицей с положительно определенной мнимой частью.

Введем, следуя [2], абелевы дифференциалы второго типа

$$
d r_{j}=\sum_{l=j}^{2 g-j+1}(l-j+1) \lambda_{l+j+1} \frac{x^{l} d x}{4 y}, \quad j=1, \ldots, g,
$$

и обозначим их матрицы $A$ - и $B$-периодов через $\eta=\left(-\oint_{A_{k}} d r_{l}\right), \eta^{\prime}=\left(-\oint_{B_{k}} d r_{l}\right)$. Оказывается, существует такая симметрическая $g \times g$-матрица $a$, что $\eta=2 a \omega$.

Обозначим через $T^{g}$ тор $\mathbb{C}^{g} /\left(\mathbb{1}_{g} \oplus \tau\right)$, изоморфный якобиану кривой (1). Пусть $\mathscr{D}=\left\{x_{1}, \ldots, x_{g}\right\}-\left\{g x_{0}\right\}$ - дивизор и $\mathscr{A}(\mathscr{D})=u=\left(u_{1}, \ldots, u_{g}\right)-$ его образ в $T^{g}$ под действием отображения Абеля $\mathscr{A}$, где

$$
u_{k}=\sum_{j=1}^{g} \int_{x_{0}}^{x_{j}} \frac{x^{k-1} d x}{y}, \quad k=1, \ldots, g,
$$

$x_{0} \in V$ - произвольная точка.

ОПРЕДЕлЕНИЕ. Гиперэллиптическая $\sigma$-функиия Клейна определяется по формуле

$$
\sigma(u)=C e^{u^{T} a u} \theta\left((2 \omega)^{-1} u-K_{x_{0}} \mid \tau\right),
$$

где $a=(2 \omega)^{-1} \eta-$ симметрическая $g \times g$-матрица,

$$
\theta(v \mid \tau)=\sum_{m \in \mathbb{Z}^{g}} \exp \pi i\left\{m^{T} \tau m+2 v^{T} m\right\}
$$

- стандартная тэта-функция, $K_{x_{0}}$ - вектор римановых констант с базисной точкой $x_{0}$ и $C$ - некоторая постоянная.

При $g=1$ функция (2), для которой $\lambda_{2}=0, \lambda_{1}=-g_{2}$ и $\lambda_{0}=-g_{3}$, есть стандартная $\sigma$-функция Вейерштрасса. Лемма (см., например, $[2,8]) .2 g \times 2 g$-матрича $\mathscr{G}=\left(\begin{array}{cc}\omega & \omega^{\prime} \\ \eta & \eta^{\prime}\end{array}\right)$ принадле-
жит $P S p_{2 g}$, m.e.

$$
\mathscr{G}\left(\begin{array}{cc}
0 & -\mathbb{I}_{g} \\
\mathbb{I}_{g} & 0
\end{array}\right) \mathscr{G}^{T}=-\frac{\pi i}{2}\left(\begin{array}{cc}
0 & -\mathbb{I}_{g} \\
\mathbb{I}_{g} & 0
\end{array}\right) .
$$

СлЕдСТвиЕ 1 (см., например, $[2,8]$ ). Пусть $x_{0}=\infty-$ базисная точка отображения Абеля и $\left[\begin{array}{lll}\varepsilon_{1}^{(0)} & \ldots & \varepsilon_{g}^{(0)} \\ \varepsilon_{1}^{(1)} & \ldots & \varepsilon_{g}^{(1)}\end{array}\right]$ - получелая характеристика вектора римановых констант. Тогда (2) обладает следующим свойством периодичности:

$$
\sigma\left(u+2 \omega_{k}^{(l)}\right)=\exp \left\{2 \eta_{k}^{(l)^{T}}\left(u+\omega_{k}^{(l)}\right)+2 \pi i \varepsilon_{k}^{(l+1)}\right\} \sigma(u),
$$

где $l=0,1(\bmod 2)$ u $\omega_{k}^{(l)}\left(\right.$ соотв. $\left.\eta_{k}^{(l)}\right)$ есть $k$-й столбеи матриць $\omega$ (соотв. $\eta$ ) при $l=0$ u $-k$-й столбеи матрицы $\omega^{\prime}$ (соотв. $\left.\eta^{\prime}\right)$ nрu $l=1$. 
Введем следующие мероморфные на $T^{g}$ функции:

$$
\wp_{p q}=-\frac{\partial^{2} \ln \sigma(u)}{\partial u_{p} \partial u_{q}}, \quad \wp_{p q r}=-\frac{\partial^{3} \ln \sigma(u)}{\partial u_{p} \partial u_{q} \partial u_{r}}, \quad \ldots, \quad p, q, r=1, \ldots, g .
$$

Согласно [1], проблема обращения Якоби разрешается следуюшим образом:

Tеорема 1. Пусть $u \in T^{g}$. Тогда $\mathscr{A}^{-1}(u)=\left\{\left(x_{k}, y_{k}\right) \in V, k=1, \ldots g\right\}$, где $\left\{x_{k}, k=1, \ldots, g\right\}$ - множество корней уравнения

$$
x^{g}-\sum_{l=1}^{g} \wp_{l g}(u) x^{l-1}=0
$$

и $y_{k}$ вычислень по $x_{k}$ с помощью формульь

$$
y_{k}=\sum_{l=1}^{g} \wp_{l g g}(u) x_{k}^{l-1} .
$$

Для $n=g+g(g+1) / 2$ введем в пространстве $\mathbb{C}^{n}$ координаты $Q, P$, где $P=\left(P_{1}, \ldots, P_{g}\right), Q^{T}=\left(q_{1}, \ldots, q_{g}\right)$ есть $g$-мерный вектор и $g$-мерные векторы $P_{k}^{T}=\left(p_{k 1}, \ldots, p_{k g}\right)$ являются столбцами симметрической матрицы $P$. Рассмотрим симметрическую степень $(V)^{g}$ кривой $V \subset \mathbb{C}^{2}$ как подмногообразие в симметрической степени $\left(\mathbb{C}^{2}\right)^{g}$ комплексной плоскости $\mathbb{C}^{2}$.

Следуя теореме 1 , определим отображение $f: \mathbb{C}^{n} \rightarrow\left(\mathbb{C}^{2}\right)^{g}$ по формуле $f(Q, P)$ $=\left(\left\{\left(x_{k}, y_{k}\right)\right\}_{k=1}^{g}\right)$, где $x_{1}, \ldots, x_{g}$ являются корнями уравнения $x^{g}-\sum_{l=1}^{g} p_{l g} x^{l-1}$ $=0$ и $y_{k}=\sum_{l=1}^{g} q_{l} x_{k}^{l-1}$.

Введем алгебраическое многообразие $\mathscr{T}$,

$$
\mathscr{T}=\left\{(Q, P) \in \mathbb{C}^{n} \mid Q Q^{T}=\Phi\left(P_{1}, \ldots, P_{g}\right)\right\},
$$

где $\Phi\left(P_{1}, \ldots, P_{g}\right)$ есть $g \times g$-матрица

$$
\Phi\left(P_{1}, \ldots, P_{g}\right)=\Lambda_{0}+\lambda_{2 g} P_{g} P_{g}^{T}+A+A^{T},
$$

причем

$$
\begin{aligned}
& A=B\left(2 P_{g} P_{g}^{T}-2 B \sum_{k=1}^{g} P_{k} e_{k}^{T}+2 \sum_{k=1}^{g} e_{k} P_{k}^{T} B^{T}+\frac{1}{2} \Lambda_{1}\right), \\
& B=P_{g} e_{g}^{T}+\sum_{k=1}^{g-1} e_{k+1} e_{k}^{T} .
\end{aligned}
$$

Здесь $e_{k}^{T}=\left(\delta_{k 1}, \ldots, \delta_{k g}\right), \Lambda_{0}=\operatorname{diag}\left(\lambda_{0}, \ldots, \lambda_{2 g-2}\right)$ и $\Lambda_{1}=\operatorname{diag}\left(\lambda_{1}, \ldots, \lambda_{2 g-1}\right)$.

Пусть $\mathbb{C}^{n-g}$ - векторное пространство с координатами $P_{1}, \ldots, P_{g}$; определим в нем подмногообразие

$$
\mathscr{K}=\left\{\left(P_{1}, \ldots, P_{g}\right) \in \mathbb{C}^{n-g} \mid \operatorname{rank} \Phi\left(P_{1}, \ldots, P_{g}\right)=1\right\} .
$$

Очевидно, что проекция $\mathbb{C}^{n} \rightarrow \mathbb{C}^{n-g}$ индуцирует двулистное разветвленное накрытие $\pi: \mathscr{T} \rightarrow \mathscr{K}$. 
ТЕОРема 2. Ограничение отображения $f$ на $\mathscr{T}$ индуиирует отображение $\mathscr{T} \rightarrow(V)^{g}$, композииия которого с отображением Абеля $\mathscr{A}:(V)^{g} \rightarrow T^{g}$ индуиирует изоморфизм между $\mathscr{T} и T^{g}-(\theta)$, где $(\theta)$ - тэта-дивизор. При этом изоморфизме проекция $\pi$ согласуется с двулистным накрытием тором его многообразия Куммера (здесь многообразием Куммера мы называем $\left.T^{g} / \pm\right)$.

Таким образом, нами получено алгебраическое описание симметрической степени гиперэллиптической кривой $V$, которое при $g=1$ совпадает с описанием эллиптической кривой - кубики Вейерштрасса. Обратим внимание на то, что уравнение, задаюшее $\mathscr{T}$, является кубическим по вектору $P_{g}$ и квадратичным по векторам $P_{1}, \ldots, P_{g-1}$, т. е. оно представляет собой кубическое уравнение в тензорной алгебре пространства $\mathbb{C}^{g}$. В случае $g=2$ уравнение $(5)$ дает описание поверхности Куммера по Бейкеру [8].

Рассмотрим симметрическую матрицу $\wp(u)=\left(\wp_{1}(u), \ldots, \wp_{g}(u)\right)$ и вектор $\wp^{\prime}(u)$

$$
\wp_{k}(u)=\left(\begin{array}{c}
\wp_{1 k}(u) \\
\vdots \\
\wp_{g k}(u)
\end{array}\right), \quad \wp^{\prime}(u)=\frac{\partial \wp_{g}(u)}{\partial u_{g}}=\left(\begin{array}{c}
\wp_{1 g g}(u) \\
\vdots \\
\wp_{g g g}(u)
\end{array}\right), \quad k=1, \ldots, g .
$$

СлЕДСТвИЕ 2. Отображение $T^{g}-(\theta) \rightarrow \mathscr{T}$, которое переводит и в $\left(\wp^{\prime}(u), \wp(u)\right)$, униформизирует алгебраическое многообразие $\mathscr{T}$ и отображение $(u,-u) \rightarrow \wp$ униформизирует многообразие $\mathscr{K}$.

В случае $g=1$ следствие 2 описывает классическую униформизацию кривой $\left\{y^{2}=4 x^{3}+\lambda_{2} x^{2}+\lambda_{1} x+\lambda_{0}\right\} \wp$ юфункциями Вейерштрасса, а в случае $g=2$ - униформизацию поверхности Куммера двумерными ю-функциями Клейна согласно Бейкеру [8].

Авторы благодарны С. П. Новикову и И. М. Кричеверу за стимулирующее обсуждение.

\section{ЛитеРАТУРА}

1. Klein F. Math. Ann., 32, 351-380 (1888). 2. Baker H. F. Abels Theorem and the Allied Theory Including the Theory of Theta Functions. Cambridge Univ. Press, Cambridge (1897). 3. Мамфорд Д. Лекции о тэта-функциях. Мир, М. (1988). 4. McKean H. P., van Moerbeke P. Invent. Math., 30, 217-274 (1975). 5. Дубровин Б. А., Матвеев В. Б., Новиков С. П. УМН, 31, вып. 1, 55-136 (1976). 6. Бухштабер В. М., Энольский В. З. УМН, 50, вып. 1, 191-192 (1995). 7. Бухитабер В. М., Лейкин Д. В., Энольский В. З. УМН, 51, вып. 1 (1996). 8. Baker H. F. Multiply Periodic Functions. Cambridge Univ. Press, Cambridge (1907).

Московский государственный университет, механико-математический факультет, кафедра высшей геометрии и топологии Институт металлофизики НАН Украины, Киев
Поступило в редакцию 6 апреля 1995 г. 